\title{
Water surface profile analysis of open channel flows over a circular surface
}

\author{
Tran Ngoc ANH *, Takashi HOSODA ** \\ * PhD Student, Dept. of Urban Management, Kyoto Univ. (606-8501 Kyoto) \\ ** Professor, Dept. of Urban Management, Kyoto Univ. (606-8501 Kyoto)
}

\begin{abstract}
This paper describes the development of a depth averaged model of open channel flows based on a generalized curvilinear coordinate system attached to the bottom surface. The model was applied to simulate the water surface profile of flows over a circular surface. An experiment was conducted in the laboratory to validate the model. Good agreements in comparison between both steady and unsteady analysis results with observation suggest that, the model can simulate flows in open channel with highly curved channel bed.
\end{abstract}

Keywords: open channel flows, depth-averaged model, curvilinear coordinates

\section{Introduction}

In a chute spillway, the stability of the free surface is strongly dependent on the geometry of the approach channel as well as that of spillway. If there is an abrupt drop at the end of channel's bed, a free overfall or a vertical fully aerated drop occurs. This phenomenon has attracted considerable attentions of investigators ${ }^{1,2,3,4)}$ since the pioneering work of Rouse ${ }^{5)}$ due to its practical engineering use in simple flow-measuring devices ${ }^{3,4)}$. But their approaches could not be applied in case of an abrupt change of channel's bed with circular shape, accompanied with small discharge that cannot result in free overfall.

On the other hand, Dressler6) derived a more general set of one-dimensional shallow water equations in which the effect of channel bed curvature was included. Using Dressler's concepts, Sivakumaran ${ }^{7,8}$ has generalized the derivation to a two-dimensional surface. Berger and Carey ${ }^{9,10}$ improved these formulations by including the vorticity features to obtain more general equations. These studies were based on the procedure of asymptotic approximation proposed by Friedrichs ${ }^{11}$ and then extended by Keller ${ }^{12}$. However this approach is significant only when the flow is sufficient shallow and gradually varied. For this reason, these models appear to be inapplicable for the case of highly curved surface.

This deficiency can be overcome if the fundamental equations are firstly transformed into a new generalized curvilinear coordinates system attached to the bottom, and then taking integral to derive the more general form of depth-averaged equations.

This paper is therefore devoted in filling this gap, by developing a $1 \mathrm{D}$ mathematical model based on depth-averaged equations with a generalized curvilinear coordinate system attached to the bottom surface. The derivation is based on the assumption of shallow water and neglecting the internal turbulent stresses with exception that, the pressure distribution was considered as a combination of hydrostatic pressure and the effect of centrifugal force due to the curvature of the channel's bed.

The developed model was applied to calculate the water surface profile in open channel attached with circular surface at the end. To 
verify the model, an experiment was conducted to measure the water surface profile, where the model results of both steady and unsteady analysis were compared with the observation.

\section{Open Channel Flows through a Circle Surface}

\subsection{Experimental Setup}

The experiments were conducted in an open-channel main flume consisted of two straight channels joined perpendicular to each other by a step ' $\mathrm{A}$ ' at the end as described in Fig 1a. The flume was $135 \mathrm{~cm}$ long, $10 \mathrm{~cm}$ wide and $20 \mathrm{~cm}$ deep, made of a steel frame with glass in all side walls and bed which allowed the

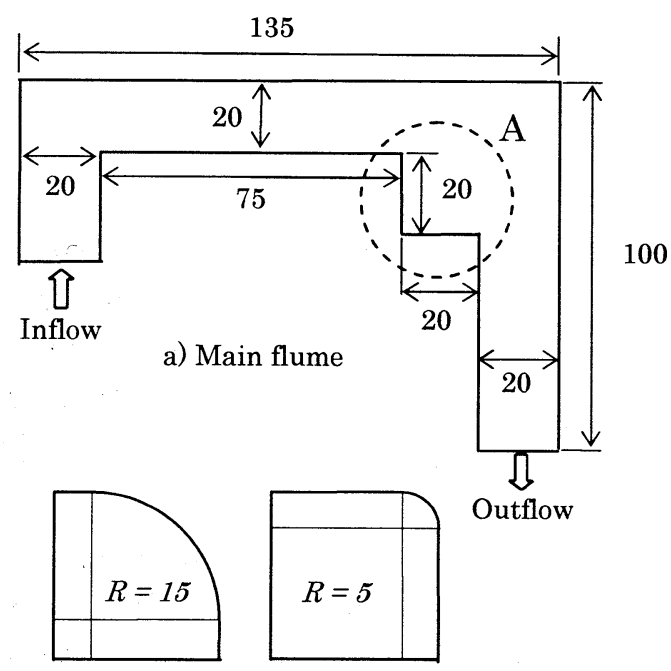

b) Part B and part C
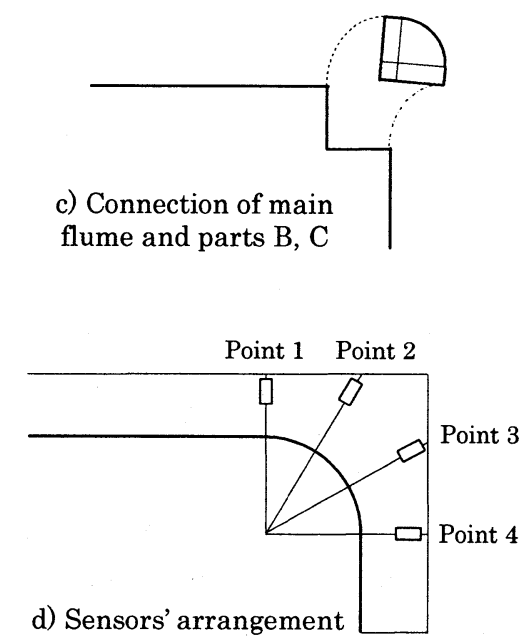

Fig. 1 Side view of the experimental facility

(All units are in centimeter) convenience for visual observations.

In this study two detachable circular parts with radius of 15 and $5 \mathrm{~cm}$ respectively were used (Fig. 1b). These parts were made such that, it is easily to be attached and detached to and from the main flume at step ' $\mathrm{A}$ ', without altering the other parts of experiment facility to form a circular channel bottom at the joint (Fig. 1c). These parts (B and $\mathrm{C}$ ) were purposely constructed to investigate the effect of different curvatures to flows. For simplicity of discussion, from now onward, the curvature with $15 \mathrm{~cm}$ and $5 \mathrm{~cm}$ radius will be referred as "large case" and "small case" respectively.

The water surface elevations were measured by the ultrasonic sensor Keyence UD-500 accompanied with the receiver Keyence NR-2000 (Fig. 2). For each case the measurements were

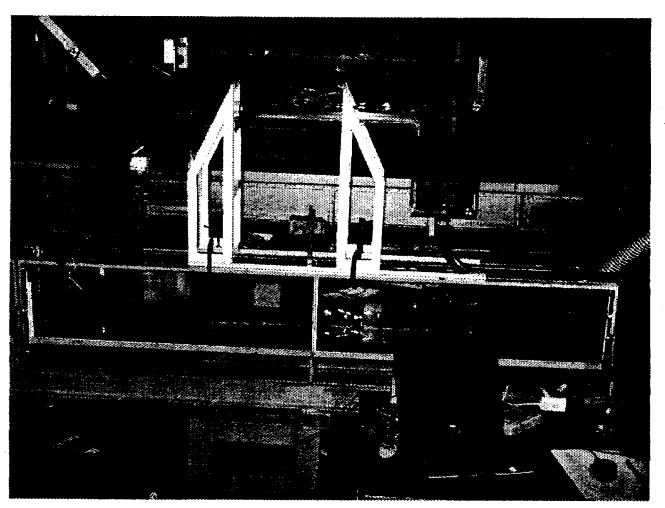

Fig. 2 Side view of the experimental site

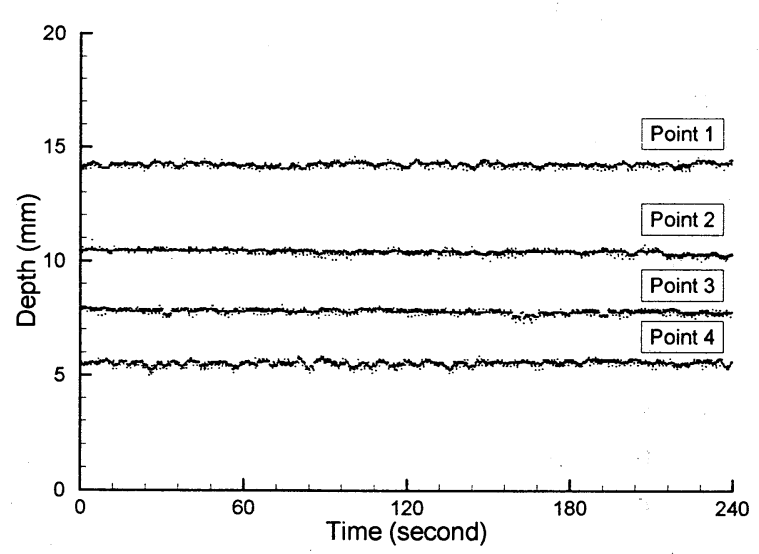

Fig. 3 An example of water depth time series at different location (TC02) 
focused in the region with the curvature of the bottom at four points $0^{\circ}$ (point 1 ), $30^{\circ}$ (point 2), $60^{\circ}$ (point 3) and $90^{\circ}$ (point 4) to the vertical direction (Fig. 1d).

\subsection{Experimental procedures}

Water was drawn from a constant head tank which is being filled by a turbine pump to ensure the steady inflow rate. The flow rate was controlled by a control valve and discharge measurements were carried out at the end of the flume for different flow conditions.

For each case, two different discharge values were investigated named TC-01 to TC-04. The samples of time series results of water depth are shown in Fig. 3. And the time-averaged values with the flow conditions are listed in Table 1.

In this paper we focused on the steady features of the water surface profile by choosing the conditions with small discharge where unsteady oscillations were not observed. Since large oscillation starts when the discharge increases, the unsteady characteristics will be investigated in the next paper.

\section{Governing Equation}

Firstly, the new generalized curvilinear coordinate is introduced. The coordinate system is generated based on the bottom plane with two axes $(\xi-\eta)$ attached to the plane and the other axis $(\zeta)$ is a straight line perpendicular to it (Fig. 4).

In Fig. $4, \eta$ is the straight axis normal to plane $(x O z)$ or is identical with $y$-axis. The surface is expressed mathematically by the equation $\zeta(x, y, z)=0$ and the derivatives of spatial independent variables $\left(x_{\xi}, x_{\eta}, x_{\zeta}, y_{\xi}, y_{\eta}, y_{\zeta}, \ldots\right)$ are denoted by the following equations:

$$
\begin{aligned}
& x_{\xi}=\frac{\eta_{y} \zeta_{z}-\eta_{z} \zeta_{y}}{J}, x_{\eta}=\frac{\zeta_{y} \xi_{z}-\zeta_{z} \xi_{y}}{J}, x_{\zeta}=\frac{\xi_{y} \eta_{z}-\xi_{z} \eta_{y}}{J} \\
& y_{\xi}=\frac{\eta_{z} \zeta_{x}-\eta_{x} \zeta_{z}}{J}, y_{\eta}=\frac{\zeta_{z} \xi_{x}-\zeta_{x} \xi_{z}}{J}, y_{\zeta}=\frac{\xi_{z} \eta_{x}-\xi_{x} \eta_{z}}{J} \\
& z_{\xi}=\frac{\eta_{x} \zeta_{y}-\eta_{y} \zeta_{x}}{J}, z_{\eta}=\frac{\zeta_{x} \xi_{y}-\zeta_{y} \xi_{x}}{J}, z_{\zeta}=\frac{\xi_{x} \eta_{y}-\xi_{y} \eta_{x}}{J}
\end{aligned}
$$

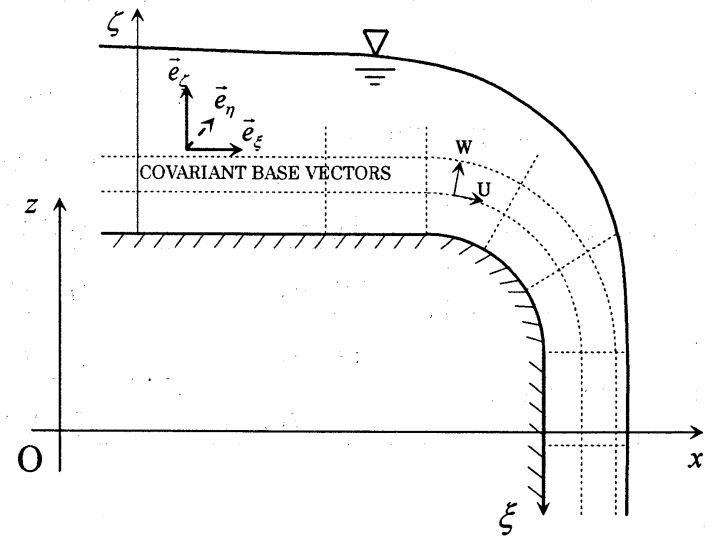

Fig. 4 Curvilinear coordinates attached to the bottom

where: $x, y, z$ are the Cartesian coordinates, subscripts indicate differentiation and the transformation Jacobian $(J)$ is defined as:

$J=\left|\begin{array}{lll}\xi_{x} & \xi_{y} & \xi_{z} \\ \eta_{x} & \eta_{y} & \eta_{z} \\ \zeta_{x} & \zeta_{y} & \zeta_{z}\end{array}\right|$

Using the assumption of constant-density, shallow water and incompressible flows, the continuity equation and Navier-Stokes equations are transformed into the new generalized curvilinear coordinate system. Taking integral from bottom to free surface with respect to $\zeta$-axis imposing the kinematic boundary condition, the depth-averaged equations are derived as follows ${ }^{13,14)}$ :

Continuity equation:

$\frac{1}{J_{0}} \frac{\partial h}{\partial t}+\frac{\partial}{\partial \xi} \frac{M}{J_{0}}+\frac{\partial}{\partial \eta} \frac{N}{J_{0}}=0$

Momentum equation in $\xi$-direction:

$$
\begin{aligned}
& \frac{\partial}{\partial t}\left(\frac{M}{J_{0}}\right)+\frac{\partial}{\partial \xi}\left(\frac{U M}{J_{0}}\right)+\frac{\partial}{\partial \eta}\left(\frac{V M}{J_{0}}\right)+\frac{1}{J_{0} h}\left(M^{2} \Gamma_{0 \xi \xi \xi}^{\xi}\right. \\
& \left.+M N \Gamma_{0 \xi \eta}^{\xi}+N M \Gamma_{0 \eta \xi}^{\xi}+N^{2} \Gamma_{0 \eta \eta}^{\xi}\right)=\frac{h}{J_{0}} G^{\xi} \\
& -\frac{\xi_{x 0}^{2}+\xi_{y 0}^{2}+\xi_{z 0}^{2}}{J_{0}} \frac{\partial}{\partial \xi} \int_{0}^{h} \frac{p}{\rho} d \zeta-\frac{\tau_{b}^{\xi}}{\rho J_{0}} \\
& -\frac{\xi_{x 0} \eta_{x 0}+\xi_{y 0} \eta_{y 0}+\xi_{z 0} \eta_{z 0}}{J_{0}} \frac{\partial}{\partial \eta} \int_{0}^{h} \frac{p}{\rho} d \zeta
\end{aligned}
$$


Table 1. Experimental conditions

\begin{tabular}{|c|c|c|c|c|c|c|}
\hline \multirow{2}{*}{ Experiment } & \multirow{2}{*}{$\begin{array}{c}\text { Discharge } \\
(\text { litter/s) }\end{array}$} & \multicolumn{4}{c|}{ Water depth (mm) } \\
\cline { 4 - 7 } & & Point 1 & Point 2 & Point 3 & Point 4 \\
\hline \multirow{2}{*}{ Small case } & TC -01 & 0.385 & 9.47 & 6.72 & 4.46 & 3.05 \\
\cline { 2 - 7 } & TC -02 & 0.775 & 14.41 & 10.51 & 7.83 & 5.58 \\
\hline \multirow{3}{*}{ Large case } & TC -03 & 0.699 & 15.53 & 8.35 & 4.31 & 3.23 \\
\cline { 2 - 7 } & TC -04 & 0.992 & 18.51 & 10.64 & 6.97 & 5.06 \\
\hline
\end{tabular}

Momentum equation in $\eta$-direction:

$\frac{\partial}{\partial t}\left(\frac{N}{J_{0}}\right)+\frac{\partial}{\partial \xi}\left(\frac{U N}{J_{0}}\right)+\frac{\partial}{\partial \eta}\left(\frac{V N}{J_{0}}\right)+\frac{1}{J_{0} h}\left(M^{2} \Gamma_{0 \xi \xi}^{\eta}\right.$

$\left.+M N \Gamma_{0 \xi \eta}^{\eta}+N M \Gamma_{0 \eta \xi}^{\eta}+N^{2} \Gamma_{0 \eta \eta}^{\eta}\right)=\frac{h}{J_{0}} G^{\eta}$

$-\frac{\xi_{x 0} \eta_{x 0}+\xi_{x 0} \eta_{y 0}+\xi_{z 0} \eta_{z 0}}{J_{0}} \frac{\partial}{\partial \xi} \int_{0}^{h} \frac{p}{\rho} d \zeta-\frac{\tau_{b}^{\eta}}{\rho J_{0}}$

$-\frac{\eta_{x 0}^{2}+\eta_{y 0}^{2}+\eta_{z 0}^{2}}{J_{0}} \frac{\partial}{\partial \eta} \int_{0}^{h} \frac{p}{\rho} d \zeta$

Where:

$\xi, \eta, \zeta:$ curvilinear coordinates

$t:$ time

$h: \quad$ the flow depth in $\zeta$-direction

$U, V:$ contravariant components of velocity vector;

$J_{0}: \quad$ Jacobian at the bottom surface

$p:$ pressure

$\rho:$ density of water

$G^{\xi}, G^{\eta}, G^{\zeta}$ : contravariant components of gravitational vector

$\tau_{b}^{\xi}, \tau_{b}^{\eta}$ : contravariant components of shear stress acting on the bottom respectively.

$\Gamma_{j k}^{i}$ : Riemann-Christoffel symbols of metric tensors expressed as:

$$
\Gamma_{j k}^{i}=-\frac{\partial x^{m}}{\partial \xi^{j}} \frac{\partial}{\partial \xi^{k}}\left(\frac{\partial \xi^{i}}{\partial x^{m}}\right), \quad(m=1,2,3)
$$

and

$M=U h, N=V h$

(The subscript zero " 0 " is denoted for the values right at the bottom plane)
The expression for pressure term is derived through the integration of momentum equation in $\zeta$-direction neglecting the acceleration and shear stress (eq. 6):

$\int_{0}^{h} \frac{p}{\rho} d \zeta=\left\{U U \Gamma_{0 \xi \xi}^{\zeta}+V U \Gamma_{0 \eta_{\xi}}^{\zeta}+U V \Gamma_{0 \xi \eta}^{\zeta}+V V \Gamma_{0 \eta \eta}^{\zeta}-G^{\zeta}\right\} \frac{h^{2}}{2}$

Neglecting transversal transport, then it follows that:

$\frac{1}{J_{0}} \frac{\partial h}{\partial t}+\frac{\partial}{\partial \xi} \frac{M}{J_{0}}=0$

$\frac{\partial}{\partial t}\left(\frac{M}{J_{0}}\right)+\frac{\partial}{\partial \xi}\left(\frac{U M}{J_{0}}\right)+\frac{1}{J_{0} h} M^{2} \Gamma_{0 \xi \xi}^{\xi}=\frac{h}{J_{0}} G^{\xi}$

$-\frac{\xi_{x 0}^{2}+\xi_{z 0}^{2}}{J_{0}} \frac{\partial}{\partial \xi}\left[\frac{M^{2}}{2} \Gamma_{0 \xi \xi}^{\zeta}-\frac{G^{\zeta} h^{2}}{2}\right]-\frac{\tau_{b}^{\xi}}{\rho J_{0}}$

\section{Steady Analysis of Water Surface Profile}

Using the system of equations (7-8); we can analyze the water surface profile of the flow over a circular surface as depicted in figure 4 . Consider the steady state, eqs. $(7-8)$ can be recast as following:

Continuity equation:

$\frac{d}{d \xi} \frac{M}{J_{0}}=0 \Leftrightarrow \frac{\mathrm{M}}{\mathrm{J}_{0}}=$ const. $=Q_{0} \Leftrightarrow M=Q_{0} J_{0}$

or $U h=Q_{0} J_{0} \Leftrightarrow U=\frac{Q_{0} J_{0}}{h}$

Momentum equation: 
$\frac{d}{d \xi}\left(\frac{U M}{J_{0}}\right)+\frac{1}{J_{0} h} M^{2} \Gamma_{0 \xi \xi}^{\xi}=\frac{h}{J_{0}} G^{\xi}$

$-\frac{\xi_{x 0}^{2}+\xi_{z 0}^{2}}{J_{0}} \frac{d}{d \xi}\left[\frac{M^{2}}{2} \Gamma_{0 \xi \xi}^{\zeta}-\frac{G^{\zeta} h^{2}}{2}\right]-\frac{\tau_{b}^{\xi}}{\rho J_{0}}$

Substitute eqs. (9), (10) into (11), after some manipulations, the equation for water surface profile can be obtained in the form of eq. (12):

$$
\frac{d h}{d \xi}=-\frac{f_{1}(h, \xi)}{f_{2}(h, \xi)}
$$

with

$$
\begin{aligned}
& f_{1}(h, \xi)=\frac{\xi_{x 0}^{2}+\xi_{z 0}^{2}}{2} \frac{d G^{\zeta}}{d \xi} h^{4}+G^{\xi} h^{3} \\
& -\frac{\xi_{x 0}^{2}+\xi_{z 0}^{2}}{2} Q_{0}^{2} J_{0}\left[J_{0} \frac{d \Gamma_{0 \xi \xi}^{\zeta}}{d \xi}+2 \Gamma_{0 \xi \xi}^{\zeta} \frac{d J_{0}}{d \xi}\right] h^{2} \\
& -Q_{0}^{2} J_{0}\left[\frac{d J_{0}}{d \xi}+J_{0} \Gamma_{0 \xi \xi}^{\xi}\right] h-f Q_{0}^{2} J_{0}^{2}
\end{aligned}
$$

and

$$
f_{2}(h, \xi)=\left(\xi_{x 0}^{2}+\xi_{z 0}^{2}\right) G^{\zeta} h^{3}+Q_{0}^{2} J_{0}^{2}
$$

The common method of analysis including singular point analysis ${ }^{13,14)}$ is applied to calculate the water surface profile in both the upstream and downstream direction from this point. The singular point is defined as the point at which both functions $f_{1}(h, \xi)$ and $f_{2}(h, \xi)$ in equation (12) are equal to zero.

The equations $f_{1}(h, \xi)=0$ and $f_{2}(h, \xi)=0$ express the quasi-normal depth line and critical depth line respectively (Fig. 5).

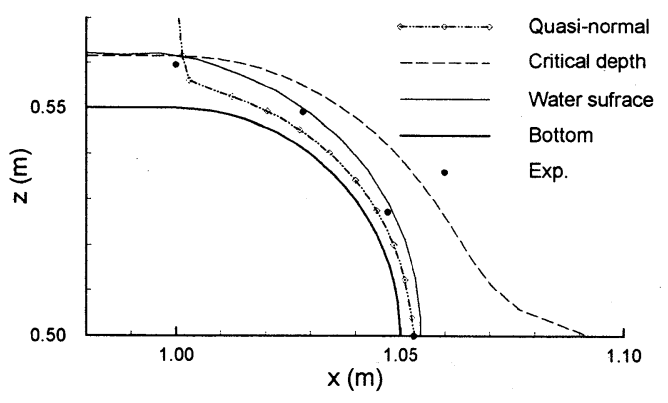

Fig. 5 Illustration of computed water surface with quasi-normal and critical depth lines (TC-01)

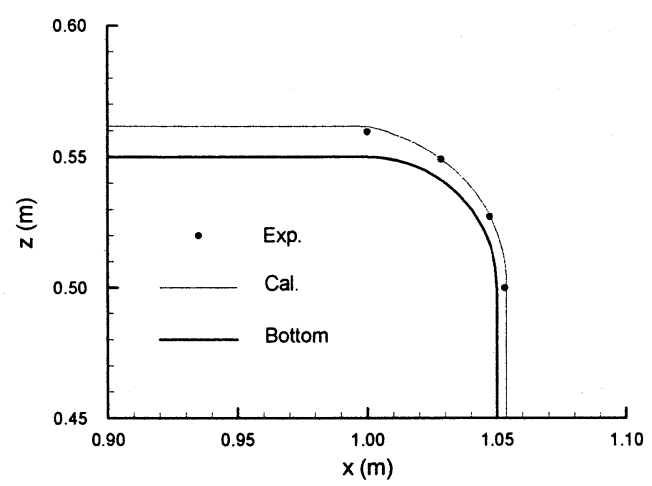

Fig. 6 Steady water surface profile with condition of TC-01

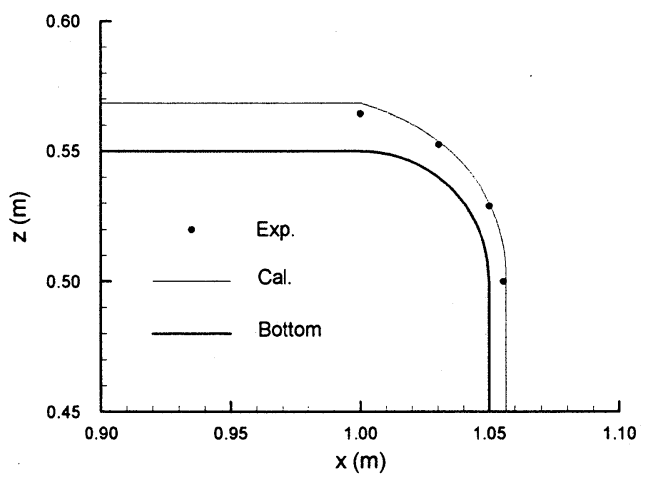

Fig. 7 Steady water surface profile with condition of TC-02

The water surface profile is derived from eq. (12) by using fourth-order Runge-Kutta scheme with the initial slope near the singular point defined by the following equation:

$$
\left.\frac{d h}{d \xi}\right|_{S}=\frac{-\left(\left.\frac{\partial f_{2}}{\partial \xi}\right|_{S}+\left.\frac{\partial f_{1}}{\partial h}\right|_{S}\right) \pm \sqrt{\left(\left.\frac{\partial f_{2}}{\partial \xi}\right|_{S}+\left.\frac{\partial f_{1}}{\partial h}\right|_{S}\right)^{2}-\left.\left.4 \frac{\partial f_{2}}{\partial h}\right|_{S} \frac{\partial f_{1}}{\partial \xi}\right|_{S}}}{\left.2 \frac{\partial f_{2}}{\partial h}\right|_{S}}
$$

(The subscript " $\mathrm{s}$ " refer to the derivatives at a singular point.)

Figures 6 to 9 show the comparison of water surface profile between the $1 \mathrm{D}$ model's results under steady state condition and the experiment for TC-01 to TC04.

It is observed from the figures that the calculated results are in consistent with the experimental data in both large and small cases. 
Note that during the experiment, because of limitation of sensors the measurements were only focused in the vicinity of the circular surface.

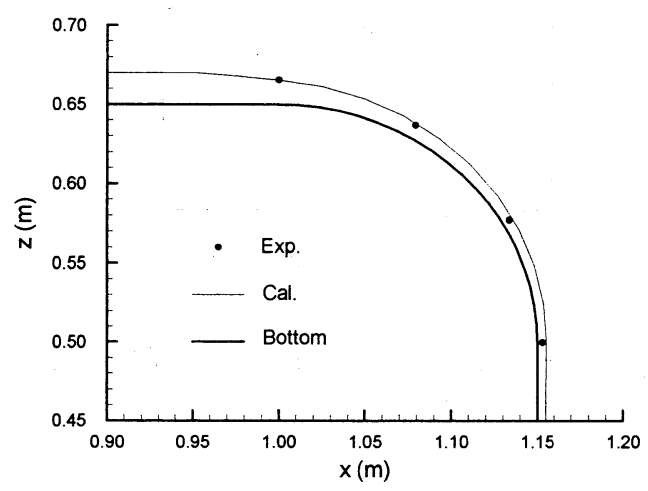

Fig. 8 Steady water surface profile with condition of TC-03

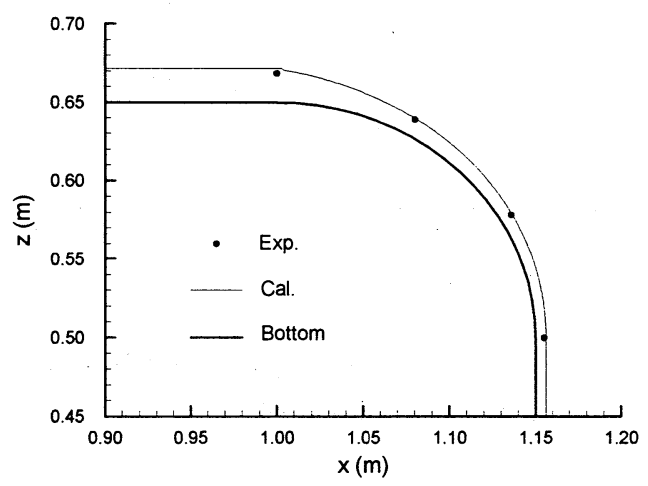

Fig. 9 Steady water surface profile with condition of TC-04

\section{Numerical Method for Unsteady Analysis}

In order to study the unsteady characteristics of flows in the next stage of this study, we tried to develop the numerical method for unsteady analysis by applying the common method used in the field of the numerical hydraulics.

For solving equations (7-8) numerically, the two-point forward scheme was used for time integration, on the other hand the First Upwind scheme was employed for the convective term based on cell-centered staggered grid (Fig. 10) for spatial discretization (eqs. 15-16).

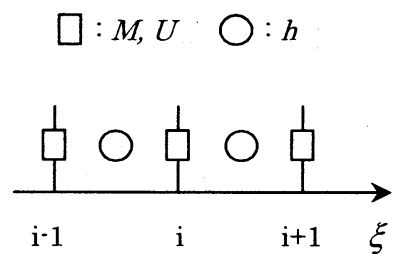

Fig. 10 Illustration of staggered grid

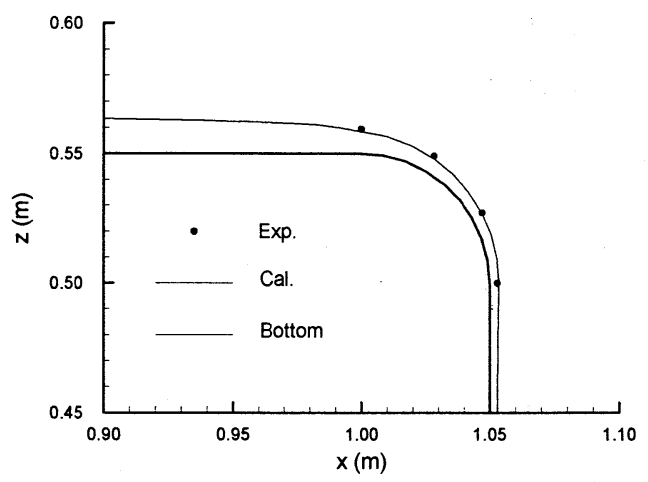

Fig.11 Water surface profile at equilibrium state of TC-01

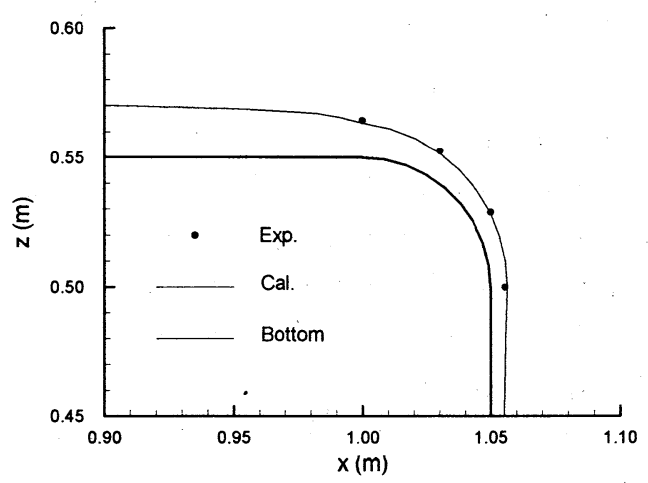

Fig. 12 Water surface profile at equilibrium state of TC-02

Continuity equation:

$\frac{1}{J_{0 i+1 / 2}} \frac{h_{i+1 / 2}^{n+1}-h_{i+1 / 2}^{n}}{\Delta t}+\frac{1}{\Delta \xi}\left[\frac{M_{i+1}^{n}}{J_{0 i+1}}-\frac{M_{i}^{n}}{J_{0 i}}\right]=0$

Momentum equation:

$$
\begin{aligned}
& \frac{M_{i}^{n+1}-M_{i}^{n}}{\Delta t}+\frac{J_{0 \mathrm{i}}}{\Delta \xi}\left[\frac{U_{i+1 / 2} M_{i+a}}{J_{0 \mathrm{i}}}-\frac{U_{i-1 / 2} M_{-1 i+b}}{J_{0 \mathrm{i}-1}}\right] \\
& +U_{i}^{2} h_{i} \Gamma_{0 \xi \xi_{i}}^{\xi}=h_{i} G_{i}^{\xi} \\
& -\frac{\left(\xi_{x 0}^{2}+\xi_{z 0}^{2}\right)_{i}}{2 \Delta \xi}\left[M_{i}^{2} \Gamma_{0 \xi \xi_{i}}^{\zeta}-M_{i-1}^{2} \Gamma_{0 \xi \xi i-1}^{\zeta}\right]
\end{aligned}
$$




$$
+\frac{\left(\xi_{x 0}^{2}+\xi_{z 0}^{2}\right)_{i}}{2 \Delta \xi}\left[G_{i}^{\zeta} h_{i+1 / 2}^{2}-G_{i-1}^{\zeta} h_{i-1 / 2}^{2}\right]-\left(\frac{\tau_{b}^{\xi}}{\rho}\right)_{i}
$$

where:

$a=\left\{\begin{array}{l}0 \text { if } U_{i+1 / 2} \geq 0 \\ 1 \text { if } U_{i+1 / 2}<0\end{array} ; \quad b=\left\{\begin{array}{l}0 \text { if } U_{i-1 / 2} \geq 0 \\ 1 \text { if } U_{i-1 / 2}<0\end{array}\right.\right.$

and $U_{i+1 / 2}^{n}=\frac{U_{i+1}^{n}+U_{i}^{n}}{2}$

The bottom shear stress was evaluated using the following equation ${ }^{13,14)}$ :

$$
\frac{\tau_{b}^{\xi}}{\rho}=f|\vec{U}| U=f\left|\vec{e}_{\xi}\right| U^{2}
$$

where $f$ is the resistant coefficient. In this study, since all walls were made of smooth glass, the value of $f=0.01$ was used. $\vec{e}_{\xi}$ is covariant base vector as shown in Fig. 4.

The model has been applied in estimating the

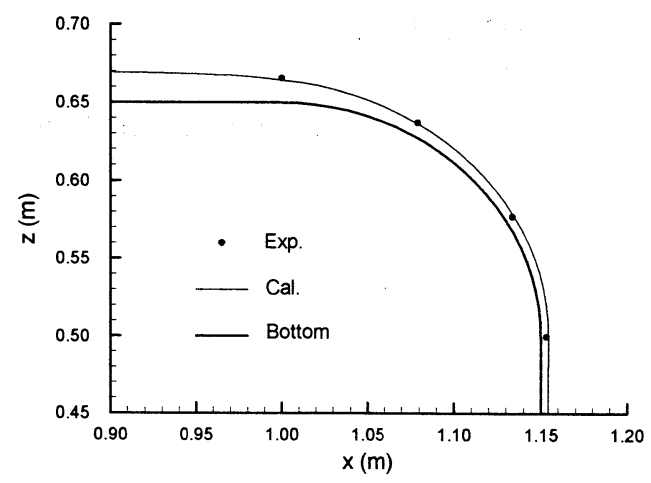

Fig.13 Water surface profileat equilibrium state of TC-0 3

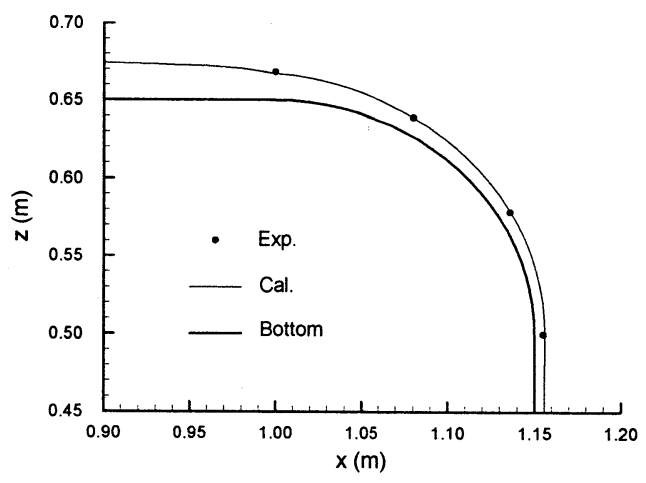

Fig.14 Water surface profile at equilibrium state of TC-04 water surface profile of flows over a circular surface Fig. 4, based on the flow conditions identical with that of the experiments (Table 1).

The inflow discharge is given at the upstream and the Neumann derivatives are provided at the downstream end to complete the boundary conditions.

In this paper, setting the constant discharge at upstream end with constant initial depth, the equilibrium state of flow was reached after around 20 seconds. Then, comparisons were made between the numerical and experimental water surface profile as shown in Fig. 11 - 12 for "small case" and Fig. 13 - 14 for "large case". In both cases, the good agreements can be observed from the figures implying the ability of the model in simulating the flows over a circular structure.

\section{Conclusion}

A simple method for computing the water surface profile of flows over a circular structure has been derived based on depth-averaged equations in a coordinate system attached to the bottom. The method was applied to calculate water surface profiles for the flows over circular structure. The experiment was conducted in the laboratory and the results showed good agreement with both the steady and unsteady analysis results.

The use of the new generalized curvilinear coordinate system during the integration process allows us to investigate the flows over a very steep channel bed or with highly curved bed, which other model can not deal with.

\section{Acknowledgment}

Authors wish to thank Mr. S. Senba who made the experimental setup. His work is highly appreciated for the success of this research.

This research is financially supported by the Ministry of Education, Culture, Sports, Science and Technology, Japan (Monbukagakusho).

\section{References}

1. Davis, A. C., Ellett, B. G. S. and Jacob, R. P.: Flow measurement in sloping channels with rectangular free overfall. J. Hydr. 
Engrg., ASCE, Vol. 124, No 7, pp. 760-763, 1998.

2. Dey, S.: End depth in circular channels. J. Hydr. Engrg., ASCE, Vol. 124, No 8, pp. 856-862, 1998.

3. Dey, S.: Free overfall in inverted semicircular channels. J. Hydr. Engrg., ASCE, Vol. 129, No 6, pp. 438-447, 2003.

4. Guo, Y.: Numerical modeling of free overfall. J. Hydr. Engrg., ASCE, Vol. 131, No 2, pp. 134-138, 2005.

5. Rouse, H.: Discharge characteristic of the free overfall. Civ. Engrg. ASCE, Vol. 6, No. 4, pp. 257-260, 1936

6. Dressler, R.F.: New nonlinear shallow-flow equations with curvature. J. Hydraul. Res., Vol 16, pp. 205-222, 1978.

7. Sivakumaran, N.S, Hosking, R.J. and Tingsanchali, T.: "Steady shallow flow over a spillway", J. Fluid Mech., Vol. 111, pp.411-420, 1981.

8. Sivakumaran, N.S, Tingsanchali, T. and Hosking, R.J.: "Steady shallow flow over curved bed", J. Fluid Mech., Vol. 128, pp.469-487, 1983.
9. Berger, R.C. and Carey, G.F: Free-surface flow over curved surfaces, Part I: Perturbation analysis. Int. J. Numer. Meth. Fluids. Vol 28, pp. 191-200, 1998.

10. Berger, R.C. and Carey, G.F: Free-surface flow over curved surfaces, Part II: Computational model. Int. J. Numer. Meth. Fluids. Vol 28, pp. 201-213, 1998.

11. Friedrichs, K.O.: Appendix. On the derivation of the shallow water theory. Comm. Appl. Math., 1, pp. 81-87, 1948

12. Keller, J.B.: The solitary wave and periodic waves in shallow water. Comm. Appl. Math., 1, pp. 323-339, 1948

13. Hosoda, T.: Depth averaged model of open channel flows over an arbitrary surface. Proceedings of the Inter. Symposium on Shallow Flows, Delf, Netherland, Part II, pp.267-271, June 16-18, 2003

14. Anh, T.N. and Hosoda, T.: Free surface profile analysis of flows with air-core vortex. J. Appl. Mech. JSCE, Vol. 7, No.2, pp.1061-1068, 2004

(Received:April 15, 2005) 